\title{
LOS DERECHOS HUMANOS DE LOS NIÑOS Y NIÑAS CON DISCAPACIDAD QUE NO TIENEN CUIDADOS PARENTALES O ESTÁN EN RIESGO DE PERDERLOS
}

\author{
THE HUMAN RIGHTS OF CHILDREN WITH DISABILITIES \\ WHO DO NOT HAVE PARENTAL CARE OR ARE AT RISK \\ OF LOSING PARENTAL CARE
}

\author{
Renata Anahí Bregaglio Lazarte* \\ Renato Antonio Constantino Caycho ${ }^{* *}$
}

\section{Resumen}

El presente documento plantea la necesidad de incorporar un enfoque de discapacidad en la regulación relativa a protección de niños y niñas con discapacidad en riesgo de perder cuidados parentales o sin ellos. Para ello, a partir de la interseccionalidad entre niñez y discapacidad se identifican los ámbitos de ejercicio de derechos por parte de estos niños y niñas con discapacidad que requieren de la incorporación de dicho enfoque y se proponen medidas.

Palabras clave: niñez, discapacidad, cuidados parentales, interseccionalidad, institucionalización.

\begin{abstract}
:
This paper raises the need of assessing a disability perspective in the Peruvian regulation related to children who lack parental care or who is under risk of losing it. To aim this objective, the authors develop the concept of intersectionality between children and disability and then identify the different excercise rights by these children that require the incorporation of this approach where is necessarily to adopt new measures.
\end{abstract}

Keywords: childhood, disability, parental care, intersectionality, institutionalization.

* Abogada y Magíster en Derechos Humanos por la Pontificia Universidad Católica del Perú (PUCP). Máster en Derechos Fundamentales por la Universidad Carlos III de Madrid. Docente Asociada del Departamento de Académico de Derecho de la PUCP. Coordinadora del Grupo Interdisciplinario de Investigación en Discapacidad de la PUCP (GRIDIS).

** Abogado y Magíster en Derechos Humanos por la Pontificia Universidad Católica del Perú (PUCP). LLM in International Legal Studies por American University - Washington College of Law. Docente del Departamento de Académico de Derecho de la PUCP. Miembro del Grupo Interdisciplinario de Investigación en Discapacidad de la PUCP (GRIDIS). 


\section{INTRODUCCIÓN}

Desde una perspectiva jurídica, la niñez es un estado de indefensión en el que se pueden atravesar una serie de violaciones a los derechos humanos. Esta es una de las razones por las que el papel de la familia es fundamental. La familia será un espacio que proteja, nutra y eduque al niño para que pueda desarrollarse en la sociedad de manera autónoma. La crianza quizá nunca sea perfecta pero es, definitivamente, un espacio en el cual niños y niñas pueden adquirir las herramientas sociales, anímicas, emocionales y culturales para enfrentarse al mundo.

Es por esto que, cuando la familia no está presente o es negligente en su labor de crianza, se espera que el Estado intervenga para contrarrestar esta situación. Las intervenciones serán diversas, según el contexto que rodea cada caso específico. Por ello, la institucionalización de un niño o niña ${ }^{1}$ ante la desprotección de su familia nuclear no debe ser considerada la respuesta automática. Este es el espíritu del artículo 9 de la Convención sobre los Derechos del Niño al disponer que:

Los Estados Partes velarán porque el niño no sea separado de sus padres contra la voluntad de éstos, excepto cuando, a reserva de revisión judicial, las autoridades competentes determinen, de conformidad con la ley y los procedimientos aplicables, que tal separación es necesaria en el interés superior del niño. Tal determinación puede ser necesaria en casos particulares, por ejemplo, en los casos en que el niño sea objeto de maltrato o descuido por parte de sus padres o cuando éstos viven separados y debe adoptarse una decisión acerca del lugar de residencia del niño.

Por ello, el acogimiento en un establecimiento distinto a la familia debe darse únicamente como una medida extrema cuando no sea posible reubicar al niño o niña en la familia extensa o en otro ámbito familiar. La institucionalización obliga a niños y niñas a vivir fuera de la sociedad, lo que les genera severos problemas para interactuar con sus pares y con otras personas. Pero además, la realidad de los niños institucionalizados es nefasta a nivel mundial (Human Rights Watch, 2014; Human Rights Watch, 2016).

En esa línea, en el Perú, la Ley 27337, Código de los Niños y Adolescentes (CNA), establece en su artículo 8 que niños y niñas tienen derecho a vivir,

El presente texto utiliza el término "niño" de acuerdo con la definición de la Convención sobre Derechos del Niño, que establece que es niño quien tiene menos de 18 años. Adicionalmente, este texto tratará de reconocer que las diferencias de género se trasladan al lenguaje e intentará hacer incidencia en el uso del género femenino de los sustantivos. 
crecer y desarrollarse en el seno de su familia. No obstante, estos podrán ser separados de su familia por circunstancias especiales definidas en la ley y con la exclusiva finalidad de protegerlos. Esta regulación legal se encuentra recogida en el Decreto Legislativo para la protección de niñas, niños y adolescentes sin cuidados parentales o en riesgo de perderlos, Decreto Legislativo $1297 .^{2}$

De manera general, se puede señalar que esta norma distingue procedimientos según se trate de situaciones de riesgo de desprotección familiar, donde se implementa un plan de trabajo individual que contempla medidas de protección (apoyo a la familia para fortalecer competencias de cuidado y crianza, acceso a servicios de educación y salud, acceso a servicios de atención especializada, apoyo psicológico a favor de niños y niñas, acceso a servicios para prevenir y abordar situaciones de violencia, o acceso a servicios de cuidado); y situaciones de desprotección familiar efectiva, que genera que el Estado asuma la tutela de niños y niñas y disponga su acogida en un centro de atención o acogida residencial (CAR) o en una familia de acogida. Esto responde al llamamiento hecho por el Comité de Derechos del Niño de Naciones Unidas que en sus Observaciones Finales al Estado Peruano en marzo de 2016, donde declaró que la normativa peruano a la fecha no distinguía claramente entre las situaciones de abandono y el riesgo (2016, párr. 47.a).

La situación de los derechos humanos de niños y niñas sin cuidados parentales suele ser bastante precaria, en particular la de aquellos que residen en CAR. De acuerdo con la Defensoría del Pueblo, en el 2011, en el Perú aproximadamente 16000 niños y niñas vivían en CAR públicos o privados (Defensoría del Pueblo, 2011, p. 27). De acuerdo con información más reciente, el Estado, a través del Programa Integral Nacional para el Bienestar Familiar (INABIF), gestiona 40 centros de acogida residencial (Defensoría del Pueblo, 2018, p. 98). En este contexto, las violaciones de derechos humanos no son aisladas. Por el contrario, reflejan la violencia que se perenniza en estos espacios y su artificialidad. Los orfanatos de antes y los centros de atención o acogida residencial de hoy mantienen estructuras que impiden la creatividad natural de los niños, que los desconectan de la realidad social en la que viven y que incluso pueden posibilitar abusos e impunidad. El Comité de Derechos del Niño, ha reportado, en Perú, casos de niños privados de alimentación o salidas como forma de castigo (2016, párr. 47.c).

Publicado en el Diario Oficial El Peruano el 30 de diciembre de 2016. Este Decreto Legislativo deroga el artículo 248 del CNA sobre supuestos para la declaración judicial de abandono; la Ley 29174, Ley General de Centros de Atención Residencial de Niñas, Niños y Adolescentes; la Ley N²6981, Ley de Procedimiento Administrativo de Adopción de Menores de Edad Declarados Judicialmente en Abandono; y la Ley N ${ }^{\circ} 30162$, Ley de Acogimiento Familiar. 
Esta situación de vulnerabilidad de los niños sin duda se ve agravada si se trata de niños o niñas con discapacidad. A nivel mundial, son niños y niñas con discapacidad quienes más sufren la institucionalización, usualmente por la prevalencia de estereotipos sobre la materia. (Mulheir, 2012 , p. 117). Esto se produce en primer lugar, porque durante años han sido alejados de sus padres o abandonados por ellos por diferentes motivos y han acabado en centros de acogida donde su desarrollo se ve severamente afectado y son más propensos a ser víctimas de violencias (Human Rights Watch, 2014). Pero en segundo lugar, porque una vez dentro de estos centros, estos no se encuentran preparados para atender sus necesidades y formarlos como sujetos activos de una sociedad a la que deben insertarse paulatinamente.

El presente artículo busca brindar algunos elementos a tomar en consideración para la incorporación del enfoque de discapacidad en las dinámicas de los centros que acogen niños que han perdido cuidados parentales.

\section{EL ENFOQUE DE DISCAPACIDAD Y LA NECESIDAD DE INCORPORARLOENELSISTEMA DE INSTITUCIONALIZACIÓN DE NIÑOS Y NIÑAS CON DISCAPACIDAD}

Desde la aparición del modelo social de la discapacidad (Oliver \& Barnes, 2012; Palacios, 2008), se ha entendido que esta no surge por una deficiencia personal sino que aparece ante la interacción de una deficiencia (de origen biológico) y una barrera (de índole social) que limita o restringe el ejercicio de derechos. Esta definición se consolida jurídicamente en el artículo 2 de la Convención sobre los Derechos de las Personas con Discapacidad (en adelante, CDPD) y en el artículo 2 de la Ley 29973, Ley General de la Persona con Discapacidad (LGPCD) ${ }^{3}$. En ese sentido, la sociedad y el Derecho tienen el deber de eliminar las barreras que impiden que estas personas puedan desarrollarse en igualdad de condiciones. A partir de esa eliminación es posible pensar en que este grupo de personas pueda desarrollarse de manera autónoma. Si bien la discapacidad es hoy por hoy un motivo de vulnerabilidad, esto no debe ser impedimento para que los niños y niñas institucionalizados puedan lograr el máximo de autonomía posible.

La autonomía individual está reconocida como principio transversal de la CDPD en su artículo 3.a y como principio rector de las políticas públicas para personas con discapacidad en el artículo 4.1.a de la LGPCD. Esta autonomía no debe ser entendida como autarquía. Tampoco como autosuficiencia (Del Águila, 2015, p. 64). Más bien debe ser entendida como una forma de ser

Publicada en el Diario Oficial El Peruano el 24 de diciembre de 2012. 
reconocido en la comunidad como un semejante que puede requerir algunas adaptaciones y apoyos para vivir en igualdad de condiciones con los demás (Constantino, 2015, p. 12).

De manera complementaria, en el caso de niños y niñas, la CDPD plantea en su artículo 3.h el principio de "respeto a la evolución de las facultades de los niños y las niñas con discapacidad y de su derecho a preservar su identidad." Lo propio hace la LGPCD en su artículo 4.1.h Esto es importante por dos razones. En primer lugar, porque implica dejar de asumir que los niños tienen una edad estandarizada para hacer ciertas cosas (sentarse, pararse, caminar, leer, hablar, etc.). En segundo lugar, a la luz del principio de autonomía ya señalado, todo niño y niña debe ser formado y educado con el objetivo de ser un miembro activo de la comunidad, sin importar qué deficiencias presenten o cuáles sean sus estándares de desarrollo. En ese sentido, niños y niñas deben ser educados respetando sus posibilidades y sin imponer parámetros normalizantes sobre ellos y ellas. A manera de ejemplo, niños y niñas sordos, antes que ser obligados aún a oralizar deberían aprender a expresarse en lengua de señas, pues lo contrario generará un retraso en sus posibilidades de expresarse adecuadamente. (Siegel, 2014).

Para lograr el efectivo disfrute de derechos de las personas con discapacidad en una sociedad (esto es, para derribar las barreras que dicha sociedad impone), la CDPD planea tres grandes medidas, que podríamos denominar como la esencia del enfoque de discapacidad: i) medidas de accesibilidad, ii) ajustes razonables y iii) reconocimiento de capacidad jurídica (Bregaglio, 2014, p. 87). La accesibilidad, de acuerdo con el artículo 9 de la CDPD son las "medidas pertinentes para asegurar el acceso de las personas con discapacidad, en igualdad de condiciones con las demás, al entorno físico, el transporte, la información y las comunicaciones, incluidos los sistemas y las tecnologías de la información y las comunicaciones, y a otros servicios e instalaciones abiertos al público o de uso público, tanto en zonas urbanas como rurales". Las definiciones en la LGPCD (artículo 15) y su Reglamento (artículo 3.1) son esencialmente similares. Por tanto, todo espacio o contexto debe poder ser usado por personas con discapacidad y esto es un requerimiento previo.

Por otro lado, los ajustes razonables son, de acuerdo con el artículo 2 de la CDPD, "las modificaciones y adaptaciones necesarias y adecuadas que no impongan una carga desproporcionada o indebida, cuando se requieran en un caso particular, para garantizar a las personas con discapacidad el goce o ejercicio, en igualdad de condiciones con las demás, de todos los derechos humanos y libertades fundamentales." La misma definición se encuentra en el Reglamento de la LGPCD (artículo 3.2). Estas medidas se realizan ex-post 
y de manera ad-hoc, por lo que no son respuestas generales sino particulares y situaciones no previstas por las normas generales (Constantino \& Galicia, 2015, p. 265).

Finalmente, el reconocimiento de la capacidad jurídica se encuentra en el artículo 12 de la CDPD. A nivel nacional, también se le encuentra en el artículo 9 de la LGPCD y en el Decreto Legislativo 1384, que modificó el Código Civil en materia de capacidad jurídica de personas con discapacidad. Como la disposición convencional plantea que este reconocimiento se dé en igualdad de condiciones, niños y niñas con discapacidad tendrán los mismos derechos que niños y niñas sin discapacidad, y deberán ser educados con miras a convertirse en personas adultas capaces de tomar decisiones importantes para sus propias vidas. Esto significará que su opinión deberá ser tomada en cuenta de la misma manera que la de quienes no tienen discapacidad y que deberán poder contar con apoyos para expresar su voluntad. ${ }^{4}$ Asimismo, cuando la institucionalización se presente como necesaria, se debe velar por la inclusión de los niños y niñas con discapacidad en dicho contexto. Ello implica que todas las acciones durante su desarrollo evolutivo deben apuntar a fortalecer sus capacidades con miras a su plena participación social y ciudadana, en igualdad de condiciones que las demás personas.

\section{MEDIDAS A TOMAR EN CUENTA PARA LA PROTECCIÓN DE NIÑOS Y NIÑAS CON DISCAPACIDAD}

A la combinación de más de un factor de discriminación se le conoce como discriminación interseccional. Este término fue acuñado por la investigadora Kimberlé Crenshaw para hablar sobre cómo las mujeres afrodescendientes sufrían una forma de discriminación que no era igual que la que sufrían las mujeres blancas ni al que enfrentaban los hombres afrodescendientes (1991). La presencia de más de una categoría de opresión en una misma persona se entiende como interseccionalidad y requiere respuestas jurídicas adecuadas.

En el caso de niños y niñas con discapacidad, la propia CDPD establece un doble enfoque a la hora de proteger sus derechos. Así, a manera de cláusula de protección general, señala en su artículo 7 lo siguiente:

\footnotetext{
Al respecto, la norma colombiana sobre capacidad jurídica de personas con discapacidad sí plantea una especificación para niños y niñas con discapacidad.

Artículo $7^{\circ}$ de la Ley 1996 de 2019.

“Niños, niñas y adolescentes. Las personas con discapacidad que no hayan alcanzado la mayoría de edad tendrán derecho a los mismos apoyos consagrados en la presente ley para aquellos actos jurídicos que la ley les permita realizar de manera autónoma y de conformidad con el principio de autonomía progresiva, o en aquellos casos en los que debe tenerse en cuenta la voluntad y preferencias del menor para el ejercicio digno de la patria potestad."
} 
1. Los Estados Partes tomarán todas las medidas necesarias para asegurar que todos los niños y las niñas con discapacidad gocen plenamente de todos los derechos humanos y libertades fundamentales en igualdad de condiciones con los demás niños y niñas.

2. En todas las actividades relacionadas con los niños y las niñas con discapacidad, una consideración primordial será la protección del interés superior del niño.

3. Los Estados Partes garantizarán que los niños y las niñas con discapacidad tengan derecho a expresar su opinión libremente sobre todas las cuestiones que les afecten, opinión que recibirá la debida consideración teniendo en cuenta su edad y madurez, en igualdad de condiciones con los demás niños y niñas, y a recibir asistencia apropiada con arreglo a su discapacidad y edad para poder ejercer ese derecho.

A partir de esta regulación general, el Estado tiene que adoptar las medidas que se necesiten para que niños y niñas con discapacidad no sean excluidos en la satisfacción de sus derechos. Adicionalmente, se menciona el concepto de interés superior del niño A partir de esta regulación general, el Estado tiene que adoptar las medidas que se necesiten para que niños y niñas con discapacidad no sean excluidos en la satisfacción de sus derechos. Adicionalmente, se menciona el concepto de interés superior del niño $[\mathrm{sic}]^{5}$ como idea clave para su protección. Finalmente, otra idea que debe rescatarse es lo referido a la participación de niños y niñas con discapacidad. Si bien ya la CDN había planteado en su artículo $12^{6}$ que niños y niñas tienen derecho a participar en lo que les incumbe, esto no siempre aplicaba a niños y niñas con discapacidad. En un contexto que busca fortalecer la autonomía de las personas con discapacidad, es necesario que esta se ejercite desde la infancia. Esto, evidentemente, tomando en cuenta la edad y la madurez del niño o niña, como manda la propia CDN. Sin embargo, es importante resaltar que, en el marco de este derecho, interpretado desde un enfoque de discapacidad, los tomadores de decisiones, no pueden excluir la opinión del niño o niñas con base en el tipo o grado de deficiencia ni a un diagnóstico. Tales consideraciones serían plenamente discriminatorias.

A partir de lo anterior, se concluye que la atención a niños y niñas con discapacidad requiere de ciertas particularidades para que su desarrollo

Entendemos que en el documento original, en inglés, se usaba el término "child" que se refiere a niños y niñas. Por tanto "niño" sería incompleto.

“Artículo 12. 1. Los Estados Partes garantizarán al niño que esté en condiciones de formarse un juicio propio el derecho de expresar su opinión libremente en todos los asuntos que afectan al niño, teniéndose debidamente en cuenta las opiniones del niño, en función de la edad y madurez del niño.

2. Con tal fin, se dará en particular al niño oportunidad de ser escuchado, en todo procedimiento judicial o administrativo que afecte al niño, ya sea directamente o por medio de un representante o de un órgano apropiado, en consonancia con las normas de procedimiento de la ley nacional." 
pueda ser igual al del resto de niños y niñas. A nivel nacional, el Decreto Legislativo 1297 dispone el apoyo integral a las niñas, niños o adolescentes con discapacidad (artículo 8). En sintonía con esta norma, en el 2012 se adoptó el "Manual de Acreditación y Supervisión de Programas para Niños, Niñas y Adolescentes Sin Cuidados Parentales" y el "Manual de Intervención en Centros de Atención Residencial de Niños, Niñas y Adolescentes Sin Cuidados Parentales ${ }^{\prime \prime}$, con el fin de estandarizar los criterios metodológicos en la atención de niños y niñas en situación de (presunto) estado de abandono. Ambos manuales identifican a niños y niñas con discapacidad como "atención especializada" y establecen criterios y estándares mínimos para garantizar una atención de calidad orientada a desarrollar destrezas para la vida independiente, así como restablecer vínculos familiares para la reinserción del niño o niña en el seno del hogar. Para lograr este objetivo será necesario tomar en cuenta medidas destinadas a lograr la igualdad, como son la accesibilidad, los ajustes razonables y el reconocimiento de la capacidad jurídica, ya mencionadas.

Es por ello que en las siguientes páginas plantearemos cómo incorporar el enfoque de discapacidad en las regulaciones del Decreto Legislativo 1297 y su reglamento. Ello corresponde en primer lugar a la situación de riesgo de perder cuidados parentales, donde será pertinente un análisis desde el Derecho de acceso a la justicia y a la familia. Pero también se analizará cómo transversalizar el enfoque de discapacidad a los derechos que debe disfrutar todo niño o niña que resida en un CAR. En este contexto, es importante señalar que de acuerdo el artículo 53.a) del Decreto 1297, cuando niños y niñas están en tutela estatal, le corresponde al Estado garantizar su "alojamiento, alimentación, educación, la salud y cuidado personal". En nuestra opinión, la principal incidencia ante la ausencia de un enfoque de discapacidad se materializaría en alojamiento (derecho a la vivienda), educación y cuidado personal. Pero consideramos también fundamental garantizar el derecho al juego, en los términos previstos en el artículo 31 de la CDN.

\subsection{DERECHO A LA JUSTICIA}

De acuerdo con el Decreto Legislativo 1297 existen dos procedimientos para atender a los NNA sin cuidados parentales: uno para NNA en riesgo de desprotección familiar, y otro para NNA en situación de desprotección efectiva. Cada uno tiene una finalidad distinta. Si bien ambos buscan incrementar los factores de protección y disminuir los factores de riesgo que incidan en la situación personal, familiar y social en la que se encuentra la NNA, en el proceso de riesgo lo hace con el fin de evitar

Aprobado mediante Resolución Ministerial 080-2012-MIMP, del 4 de abril de 2012. 
situaciones de desprotección familiar (artículo 24), mientras que el proceso de desprotección busca lograr el retorno a su familia, siempre que ello responda a su interés superior (artículo 43).

Para responder a la primera finalidad, como ya se señaló, en el proceso de riesgo de desprotección familiar, se pueden adoptar las siguientes medidas: apoyo a la familia para fortalecer competencias de cuidado y crianza, acceso a servicios de educación y salud, acceso a servicios de atención especializada, apoyo psicológico a favor de los NAA, acceso a servicios para prevenir y abordar situaciones de violencia, o acceso a servicios de cuidado. Por su parte, el proceso de desprotección familiar culmina con la disposición de que el NNA resida en un CAR o en una familia de acogida.

Uno de los aportes del Decreto Legislativo 1297, en términos de acceso a la justicia, y a la vez, de reconocimiento de capacidad jurídica a los menores (compatible con el enfoque de protección integral de la CDN), es la posibilidad que tienen niños y niñas de denunciar sus propias situaciones de riesgo o desprotección (artículo 17). En la misma línea, se les reconoce como parte en el proceso (artículo 12), lo que les permite, entre otras cosas, acceder al expediente antes de la emisión del informe de evaluación en el marco del procedimiento de desprotección (artículo 47), e impugnar como la resolución judicial emitida en el marco de dicho procedimiento (artículo 57).

Por otro lado, la norma dispone que para emitir el informe de evaluación en el marco del procedimiento de riesgo, se debe escuchar la opinión del NNA en una actuación especial (artículo 28). Esta opinión, además, debe estar explicitada y formar parte de los fundamentos de la resolución que declara si hay o no situación de riesgo. Esto, sin duda, busca cumplir con el artículo 12 de la CDN, que dispone que los NNA tendrán derecho a expresar sus opiniones libremente en todos los asuntos que lo afecten, y éstas serán debidamente tomadas en cuenta, en función de la edad y madurez de estos. Además, busca fomentar la garantía de debida motivación de las decisiones que se adoptan en el marco de procesos o procedimientos.

Ahora bien, garantizar la participación de niños y niñas es clave para que sean considerados verdaderos sujetos de derecho y no simples objetos que obedecen a los deseos de ciertos adultos. Sin embargo, para que pueda participar adecuadamente, previamente, es deseable que el menor reciba la información y asesoramiento necesario que le permita el ejercicio de este derecho en un lenguaje comprensible, en formatos accesibles y adaptados a sus circunstancias. Es en este contexto donde se vuelve necesario tener una mirada interseccional. 
Lamentablemente, la norma no señala la necesidad de brindar medidas de accesibilidad y ajustes razonables en los procedimientos para los niños y niñas con discapacidad. Si bien es posible aplicar el mandato general de accesibilidad y ajustes razonables de la $\mathrm{LGPCD}^{8}$, las posibilidades de que un operador de justicia lo haga sin estipulaciones expresas son bajas. Esto se debe principalmente a que los organismos públicos no han implementado buenas prácticas para la atención a personas con discapacidad. ${ }^{9}$ Tales implementaciones deben darse en el marco del artículo 5.g) del Decreto Legislativo 1297, que señala que niños y niñas tienen derecho a ser "ser informados de las medidas de protección dispuestas a su favor, sobre la situación de los miembros de su familia, así como del estado del procedimiento", y de manera más específica, con el artículo 99 que dispone que en la audiencia especial para el niño, el juez debe garantizar que cuente con la información y pueda expresarse de acuerdo con sus circunstancias como son la edad, la discapacidad o la lengua de origen. En ese sentido, como parte de las medidas de accesibilidad se debería proveer documentos en Braille o interpretación de lengua de señas para quienes lo requieran. También intérpretes para niños y niñas con sordoceguera y materiales para la comunicación aumentativa y adaptativa. Adicionalmente, deberán adoptarse los ajustes razonables necesarios: más tiempo para la toma de decisiones, cambios en la forma de realizar interrogatorios y creación de entornos adecuados y seguros para niños y niñas con discapacidad.

\subsection{DERECHO A LA VIDA EN COMUNIDAD: NO SEGREGACIÓN Y EXTERNAMIENTO}

Históricamente, las personas con discapacidad se han visto impedidas de vivir en la comunidad en igualdad de condiciones con el resto. En general, en muchos casos alrededor del mundo se han visto obligadas a

\footnotetext{
Al respecto, cabe señalar que la Ley 29973 - Ley General de la Persona con Discapacidad establece en su artículo 21.1 que el Estado debe garantizar la comunicación en "la lengua de señas, el sistema braille, la comunicación táctil, los macrotipos, la visualización de textos, los dispositivos multimedia, el lenguaje escrito, los sistemas auditivos, el lenguaje sencillo, los medios de voz digitalizada y otros modos y medios aumentativos o alternativos de la comunicación".

$9 \quad$ De acuerdo con el artículo 23.1 del Reglamento de la Ley General de la Persona con Discapacidad (DS 002-2014-MIMP) los organismos vinculados a la administración de justicia "garantizan la tutela preferente y accesibilidad de las personas con discapacidad a la infraestructura de los órganos que lo conforman; disponen las medidas conducentes al acceso a todos los servicios aprobados que requieran, así como la disposición de los apoyos y recursos que garanticen su seguridad, movilidad, comprensión, privacidad y comunicación. Establecen e implementan manuales de buenas prácticas de revisión permanente." De acuerdo con el artículo 23.1 del Reglamento de la Ley General de la Persona con Discapacidad (DS 002-2014-MIMP) los organismos vinculados a la administración de justicia "garantizan la tutela preferente y accesibilidad de las personas con discapacidad a la infraestructura de los órganos que lo conforman; disponen las medidas conducentes al acceso a todos los servicios aprobados que requieran, así como la disposición de los apoyos y recursos que garanticen su seguridad, movilidad, comprensión, privacidad y comunicación. Establecen e implementan manuales de buenas prácticas de revisión permanente."
} 
vivir en instituciones, orfanatos, asilos o encerrados en casas familiares sin un real contacto con el exterior. Es por eso que la CDPD agregó un artículo específico para cambiar dichas condiciones. Así, en su artículo 19 reconoce "el derecho en igualdad de condiciones de todas las personas con discapacidad a vivir de forma independiente y a ser incluidas en la comunidad, con la libertad de elegir y controlar su vida." (Comité CDPD, 2017, pár. 2). De acuerdo con el apartado a) del artículo 19, este derecho abarca el que las personas con discapacidad puedan elegir el lugar de residencia y dónde y con quién vivir y no se vean obligadas a vivir con arreglo a un sistema de vida específico.

Además, para el caso de niños y niñas con discapacidad, el Comité de Personas con Discapacidad, ha señalado que el derecho a vivir de manera independiente implica el derecho a vivir en una familia (Comité CDPD, 2017, pár. 37). Es claro que dicho estándar es imposible de cumplir en el corto plazo. Hasta que se cumpla, lo que sí debe lograrse en espacios institucionalizados con niños y niñas con discapacidad es que se desarrollen al máximo de sus capacidades. Eso quiere decir, pensando en la futura ciudadanía de estos niños y niñas, que es necesario que se les eduque pensando en que en la adultez deben vivir en la comunidad de acuerdo al sistema de vida que elijan: van a poder trabajar; entablar relaciones amicales o sentimentales; ser padres y madres; participar en cultos; votar y demás actividades que todos y todas realizamos. Es por eso que el periodo de institucionalización no debe impedir lograr esas metas. Debe ser un espacio que, con sus carencias, trabaje por la máxima autonomía de niños y niñas con discapacidad.

A nivelnacional, uno de los esfuerzos para evitar la institucionalización de niños y niñas con discapacidad ha sido la elaboración del "Manual para la Atención Integral y la Prevención del Abandono de las Personas con Discapacidad"10, que establece parámetros para la atención de las personas con discapacidad tanto en el ámbito comunitario (a fin de prever el abandono por razones de discapacidad) como en las que se encuentran institucionalizadas en los centros de atención residencial. Este es un primer esfuerzo gubernamental por estandarizar criterios de atención específicos encaminados a fortalecer capacidades comunitarias, familiares e individuales.

El Manual toma en cuenta los principios de la CDPD y busca dotar a las familias de diferentes recursos para atender personas con discapacidad.

10 Resolución de INABIF 107-2015, del 26 de febrero de 2015. 
Así, los servicios de INABIF pueden guiar a las familias hacia el acceso a políticas públicas o beneficios. También se intenta brindar servicios de cuidado hacia las personas con discapacidad. No obstante, también resulta necesario que gobiernos locales y regionales generen dichos recursos. Sin ellos, las pocas intervenciones de INABIF devienen en inútiles.

No obstante lo anterior, resulta cuestionable que niños y niñas con discapacidad severa o multidiscapacidad acogidos por INABIF residan en CAR destinados exclusivamente a personas con discapacidad. ${ }^{11}$ Si bien los CAR de INABIF señalan ser inclusivos y atender a niños y niñas con discapacidad, subsiste un sistema especializado de tres CAR para niños y niñas con discapacidades más severas y multidiscapacidad. Hasta 2017, existían 117 niños viviendo en estos CAR especializados en atención a discapacidad (INABIF, 2017, p. 13).

\section{Tabla 1}

\section{Acciones de seguimiento para la reinserción del NNA residentes con discapacidad del INABIF al 2015}

\begin{tabular}{|lccc|}
\multicolumn{1}{|c}{ CAR } & Población atendida & Visitas domiciliarias a familiares del NNA & Visitas de familiares al CAR \\
\hline Matilde Pérez Palacio & 99 & 0 & 8 \\
\hline Esperanza & 17 & 0 & 4 \\
\hline Niño de Jesús de Praga & 58 & 0 & 0 \\
\hline
\end{tabular}

Fuente: Elaboración propia. Basado en el reporte estadístico INABIF 2015.

De acuerdo con un reporte de actividades anuales de 2015, los tres CAR de INABIF con presencia exclusiva de niños o niñas con discapacidad ${ }^{12}$ realizan acciones intramurales que si bien favorecen parte del desarrollo de niños y niñas, no los preparan para reinsertarse en el entorno familiar y/o comunitario (Tabla 1). ${ }^{13}$ Así, por ejemplo, la cantidad de visitas que estos niños y niñas realizan a sus familiares es nula. Con respecto a visitas de familiares a estos niños y niñas, los números son también ínfimos. Esto puede generar una pérdida del vínculo familiar y permitir la prolongación de la estancia de niños y niñas con discapacidad residentes en la institución.

11 No se cuenta con información respecto a en donde residen niños y niñas con discapacidad en los otros CAR públicos y privados.

12 Es importante señalar que no existe información actualizada disponible respecto de la población de todos los CAR a nivel nacional, ni de las actividades realizadas por estos. La única información actualizada es la que produce INABIF respecto de los CAR que esta entidad regenta.

13 Es importante señalar que no existe información actualizada disponible respecto de la población de todos los CAR a nivel nacional, ni de las actividades realizadas por estos. La única información actualizada es la que produce INABIF respecto de los CAR que esta entidad regenta. 
Uno de los motivos que puede generar esto es la segregación que existe entre los CAR. Si bien todos ellos deberían ser inclusivos, se han señalado algunos para la atención especializada de niños y niñas con discapacidades severas o multidiscapacidad. Estos CAR se encuentran en Lima, lo que se traduce en un desarraigo y restricción de los vínculos familiares de los NNA con discapacidad que residen en el interior del país y que son declarado en estado de desprotección familiar.

La decisión de segregar a niños y niñas con discapacidad severa sigue el modelo actual de educación de niños y niñas con discapacidad en el Perú. Si bien la ley señala que la educación es inclusiva en todos los niveles, subsiste la educación especial para niños y niñas con discapacidad severa y multidiscapacidad (Constantino, Bregaglio, \& Cueva, 2016, p. 51). Esto fomenta la segregación antes que la inclusión, y resta esfuerzos al intento de promover la autonomía e inserción social de estos niños y niñas. Si ya es complejo lograr la inclusión social de niños y niñas institucionalizados, se vuelve titánico pensar que es posible lograr que niños y niñas con discapacidad institucionalizados puedan incluirse fácilmente si solamente conocen a pares en su misma situación. Esta situación requiere que, de manera progresiva y decidida, se inicie un proceso de verdadera inclusión de niños y niñas con cualquier clase de discapacidad en los CAR.

Por otro lado, es importante señalar que en los CAR de atención a personas con discapacidad, no se produce el externamiento de la persona cuando alcanza la mayoría de edad, a diferencia del resto de CAR. Esto afecta el derecho a la libertad de las personas con discapacidad que pudieran no querer permanecer en dicha institución, pero también afecta su derecho a la vida en comunidad, pues la persona no puede elegir su lugar de residencia.

En estos casos resulta importante plantear la necesidad de una mirada desde la autonomía. Los adultos tienen derecho a decidir su lugar de residencia. En caso no tengan la posibilidad de acceder a una vivienda propia o familiar, los centros de atención residencial pueden brindar dicho espacio pero teniendo en cuenta la autonomía de la persona. Esto quiere decir que no puede haber horarios rígidos o reglas excesivas para la libertad de una persona.

\subsection{DERECHO A LA FAMILIA}

A partir de la lectura de la CDN, la decisión de retirar a padres y madres la tutela de sus hijos o hijas y asignársela al Estado se entiende justificada en el interés superior del niño [sic], en la medida que estos niños 
y niñas no pueden ser criados en un ambiente nocivo. No obstante, podría considerarse violatorio de este derecho cuando un juez toma la decisión de internar a un niño o niña en un CAR, sin que esto sea estrictamente necesario (Comité de Derechos del Niño, 2016, párr. 47.b), o si es que los niños y niñas institucionalizados no mantienen relación con la familia.

Respecto a la primera, la regulación del Decreto Legislativo 1297 es positiva al eliminar la "declaración judicial de abandono" (como regulaba el CNA) y establecer un procedimiento de "declaración de riesgo o desprotección familiar", pues transita de un procedimiento enfocado en constatar la situación de abandono, a uno que considera al niño o niña en el centro de su intervención para garantizar su protección. Sin embargo, muchos jueces han tomado como premisa que la principal forma de proteger a un niño o niña es logrando su internamiento en un CAR. A manera de ejemplo, en su Informe No. 153, la Defensoría del Pueblo señaló que de un total de 122 procesos tutelares, en 82 de ellos se decretó como medida de protección el acogimiento residencial en los CAR, pese a su declarada excepcionalidad (2011, p. 142).

Este proceder contraviene lo señalado por las Directrices de Naciones Unidas sobre las Modalidades Alternativas de Cuidado de los Niños (parágrafo 20) y el Decreto Legislativo 1297 (artículo 58.d y 101), que disponen que la institucionalización en un CAR debe ser una medida de última ratio, y que siempre se debe preferir que el menor siga en contacto con su familia, incluyendo la extendida, o que sea acogido por otra familia que le permitan generar lazos de confianza y cariño.

En los casos de niños y niñas con discapacidad la institucionalización en CAR en estos contextos es frecuente. Ello no necesariamente responde a un prejuicio del operador de justicia, si no al hecho de que resulta difícil encontrar familias extensas o terceras familias que quieran acoger al niño o niña. Frente a esto, sin embargo, no debe asumirse que la única opción debe ser la institucionalización. De acuerdo con el artículo 23.5 de la Convención sobre los Derechos de las Personas con Discapacidad (CDPD): los Estados "harán todo lo posible, cuando la familia inmediata no pueda cuidar de un niño con discapacidad, por proporcionar atención alternativa dentro de la familia extensa y, de no ser esto posible, dentro de la comunidad en un entorno familiar." Asimismo, la Directriz 131 de Naciones Unidas establece que los "niños con necesidades especiales, como discapacidades, deberían poder acogerse a un sistema de asistencia apropiado, que entre otras cosas les permita eludir una institucionalización innecesaria." De esta manera, se debe evitar que niños y niñas sean enviados a CAR. Ello se enmarca en 
el derecho a vivir en familia y en comunidad, señalado claramente en la CDPD. ${ }^{14}$

Por ello, antes que optar por la institucionalización como respuesta automática, habría que pensar en las razones por las que estas familias no quieren acoger a estos niños y niñas. Sin perjuicio de que pueda desarrollarse un estudio profundo, nos atrevemos a afirmar que una de las razones serán los costos que el cuidado de un niño o niña con discapacidad pueden irrogar, tanto en asistencia médica y medicamentos, como en insumos o personal de cuidado, o en transporte. Al respecto, se sabe que, en el Perú, el Estado no provee casi ningún apoyo para la crianza de niños y niñas con discapacidad (Aguerre et al., 2019) y que, en muchos casos, las familias con miembros con discapacidad no son adecuadamente incluidos en las políticas de protección social (Bernabe-Ortiz et al., 2016). En ese sentido, y a modo de ajuste razonable, sería necesario que la política de programas de acogimiento familiar establezca una subvención adecuada a las familias que acogen niños y niñas con discapacidad, y supervisar el gasto de esta subvención de manera que sea utilizada para cubrir los sobrecostos de atención generados por la discapacidad. Al respecto, cabe señalar que la condición de discapacidad es una forma de exclusión para la obtención de beneficios en el programa JUNTOS. Así, este programa señala en la Directiva 02-2016-MIDIS /PNADP-DE "Procesos de Afiliación y Mantenimiento de Padrón de Hogares" ${ }^{15}$ que:

Los hogares que cuenten con un miembro objetivo inactivo permanente (por discapacidad severa certificada) (...) deberán contar con al menos un miembro objetivo que pueda cumplir corresponsabilidad, de lo contrario serán desafiliados por pérdida de criterio de elegibilidad. Para mantener la condición de afiliado, todo hogar debe tener al menos un miembro objetivo que pueda cumplir corresponsabilidad, caso contrario será desafiliado. ${ }^{16}$

De esta manera, la directiva excluye a las familias que tengan hijos o hijas con discapacidad severa y que no tengan otros hijos o hijas que puedan cumplir la "corresponsabilidad" (asistencia al colegio y chequeos médicos).

14 Artículo 29. b): Los Estados Partes en la presente Convención reconocen el derecho en igualdad de condiciones de todas las personas con discapacidad a vivir en la comunidad, con opciones iguales a las de las demás, y adoptarán medidas efectivas y pertinentes para facilitar el pleno goce de este derecho por las personas con discapacidad y su plena inclusión y participación en la comunidad, asegurando en especial que:

(...)

b) Las personas con discapacidad tengan acceso a una variedad de servicios de asistencia domiciliaria, residencial y otros servicios de apoyo de la comunidad, incluida la asistencia personal que sea necesaria para facilitar su existencia y su inclusión en la comunidad y para evitar su aislamiento o separación de ésta."

15 Disponible en: http://www.juntos.gob.pe/modulos/mod_legal/archivos/016-2016\%20Directiva\%20Afiliacion $\% 20 \mathrm{y} \% 20 \mathrm{Mtm}$ to $\% 20$ Padron.pdf

$16 \quad$ Artículo 8.1.13.b 
Respecto a lo segundo, es importante recordar que la atribución de tutela al Estado bajo ninguna circunstancia suprime el derecho a la familia de estos niños y niñas, tal como lo establece el artículo 9.3 de la CDN, al disponer que:

Los Estados Partes respetarán el derecho del niño que esté separado de uno o de ambos padres a mantener relaciones personales y contacto directo con ambos padres de modo regular, salvo si ello es contrario al interés superior del niño.

De igual forma, las Directrices de Naciones Unidas sobre las Modalidades Alternativas de Cuidado de los niños establecen que se debe procurar que los niños y niñas permanezcan o vuelvan a estar bajo la guarda de sus padres o, cuando proceda, de otros familiares cercanos (parágrafo 3). En ese sentido, salvo que se justifique que el contacto con la familia de origen es contrario al interés superior del niño [sic], los niños y niñas en CAR o en familias temporales, tendrán derecho a mantener contacto con sus padres. Ya para el 2010 la Defensoría del Pueblo señaló que más de la mitad de los residentes mantenían algún vínculo con sus familias (p. 244), lo que ratificaba la obligación de trabajar el proceso de reinserción familiar con las referidas familias. Así lo establece ahora el Decreto Legislativo 1927, que señala que el niño o niña en acogimiento familiar o residencial tienen derecho a mantener relaciones personales y a ser visitados por su padre, madre, otros miembros de la familia de origen o extensa y otras personas (artículo 83). Esto es importante, además, para lograr retorno de niños y niñas a sus familias de origen. El mismo decreto establece como uno de los criterios para disponer el retorno, el que se hayan preservado los vínculos familiares (artículo 94).

Sin embargo, satisfacer esta dimensión del derecho a la familia puede resultar complejo. El número de CAR disponible por provincia y departamento es dramáticamente bajo. Ello hace que, cuando un niño o niña es enviado a un CAR, pueda ser trasladado a otra ciudad, lejos de su familia de origen, lo que dificulta la posibilidad de mantener los lazos. Esto, como ya señalamos, resultaría particularmente grave en los casos de niños y niñas con discapacidad, pues los CAR públicos especializados en discapacidad severa o multidiscapacidad se encuentran únicamente en Lima. Si niños y niñas con discapacidad de provincia son trasladados a Lima, sus posibilidades de mantener el vínculo familiar se vuelven más remotas.

Si bien no existen cifras precisas que dimensionen este problema para el caso de niños y niñas con discapacidad, la Defensoría del Pueblo lo ha señalado para el caso de niños y niñas pertenecientes a minorías culturales 
en su Informe Defensorial 150. Allí reportó que un 6.7\% de los residentes, procedían de regiones distintas a la región donde se encuentran ubicados los CAR (2010, p. 237), y que el 9.5\% del total de los residentes se expresaban en una lengua materna distinta del castellano (2010, p. 238).

Otro aspecto que dificulta la satisfacción del derecho a la familia es la separación de hermanos/as, donde cada hermano acude a un distinto centro de atención residencial. Esta clase de situaciones complican la posibilidad del niño de volver a reinsertarse en su espacio familiar. Es importante señalar que el Manual de Acreditación y Supervisión de Programas para Niños, Niñas y Adolescentes Sin Cuidados Parentales ${ }^{17}$ tiene como uno de sus indicadores de estándares de calidad, el promover procesos de integración entre los hermanos que residen en diferentes CAR. Esto es particularmente riesgoso en el caso de hermanos/as donde alguno/a tenga discapacidad severa o multidiscapacidad, pues será enviado/a al CAR especializado. Por ello, sería idóneo que la resolución que dispone la institucionalización de hermanos/as contemple que esta sea en el mismo CAR, y (como ya señalamos), que todos los CAR sean plenamente inclusivos.

\subsection{DERECHO A LA EDUCACIÓN}

La importancia del derecho a la educación radica, principalmente, en su calidad de derecho "habilitante", posibilitando el ejercicio de otros derechos (Tomasevski, 2001, p. 10). Para niños y niñas, la posibilidad de acceder a una buena educación puede ser crucial para escapar del círculo vicioso de la pobreza. Incluso Naciones Unidas ha señalado que la educación es el "principal medio que permite a adultos y menores marginados económica y socialmente salir de la pobreza y participar plenamente en sus comunidades." (Comité de Derechos Económicos, Sociales y Culturales, 1999, párr. 1).

De acuerdo con el Decreto Legislativo 1297, la garantía del derecho a la educación forma parte de los dos procedimientos: el de riesgo y el de abandono. En el primer caso, se dispone la provisión de servicios de educación como una de las medidas a adoptar frente a una declaratoria de situación de riesgo (artículo 33.b). En el segundo caso, se dispone que la tutela estatal debe garantizar el derecho a la educación (artículo 53). En el caso de niños y niñas que residen en CAR, esto se traduce en asegurar la cobertura del servicio educativo (artículo 75.a). Además, en el caso de niños y niñas con discapacidad el Decreto Legislativo 1297 establece que se debe fortalecer la educación inclusiva (artículo 75.f), en consonancia con el artículo 24 de la CDPD.

17 Aprobado mediante Resolución Ministerial 081-2012-MIMP, del 4 de abril de 2012. 
En tal sentido, ubicar el centro educativo al interior del CAR, como ocurre en el caso del Puericultorio Pérez Araníbar o en el CAR Esperanza ${ }^{18}$ no sería lo más conveniente desde una perspectiva amplia del derecho a la educación y menos desde el enfoque de educación inclusiva. Si bien en el caso de niños y niñas con discapacidad, se puede alegar que evita el problema de tener que salir a una ciudad muchas veces inaccesible; la solución no debe ser que la experiencia escolar sea meramente una continuidad de lo que se vive en el CAR. La educación va más allá de la mera adquisición de conocimientos. Más bien, es un cúmulo de experiencias que permiten el desarrollo integral de la persona. En ese sentido, la participación de niños y niñas con discapacidad colabora con que el resto de la sociedad los incluya más fácilmente (Constantino et al., 2016, p. 9).

Dado que las escuelas son un espacio intermedio entre la familia y la sociedad, que proporciona un contexto para "el desarrollo de la comprensión crítica y la producción y la reproducción de la crítica social" (Walzer, 2001, p. 208), es necesario que niños y niñas que viven en CAR puedan compartir escuela con sus pares que no viven en CAR, incluso en el caso de niños y niñas con discapacidad. En tanto la educación es un proceso formativo está compuesto también por la reflexión, el debate y la vivencia con otras personas, hacer que quienes ya viven en ambientes segregados reciban una educación que refuerza esa premisa es condenarlos a aislarse del mundo.

Otro tema particularmente preocupante es el referente a la educación sexual de niños y niñas que viven en los CAR. La educación sexual es parte de los derechos sexuales y reproductivos. (Minieri, 2017). Y con mucha frecuencia, niñas con discapacidad son excluidas de la posibilidad de acceder a servicios de salud sexual y reproductiva. Esto se debe, principalmente, a la prevalencia de los estereotipos referidos a la sexualidad de personas con discapacidad. Tradicionalmente, se le ha visto como asexuados o como incapaces de comprender la sexualidad (Valega, 2016). Ante ello, la respuesta ha sido el silencio institucional y el evitar las muestras de sexualidad. En el caso de las mujeres, en particular, mientras que a las mujeres con lo sexys o atractivo, a las mujeres con discapacidad se las asocia con la idea de que son incapaces de sentir placer (Minieri, 2017, p. 25). También suele asumirse la infantilización a la mujer con discapacidad, lo que lleva a poner en entredicho su capacidad para tomar decisiones sobre su cuerpo.

En ese contexto, la educación sexual hacia las personas con discapacidad no solo debe tener fines informativos, sino también fines de reivindicación de la capacidad sexual y reproductiva de estas personas. Por ello, correspondería

18 La información proviene de visitas a estos CAR. 
que los funcionarios del CAR estén adecuadamente capacitados para impartir nociones de educación sexual a niños y niñas con discapacidad. Para ello, se necesitaría contar con materiales adecuados para la difusión, así como especialistas adecuados para la orientación a niños y niñas con discapacidad en la materia. Tales especialistas deben reconocer la agencia y la sexualidad de niños y niñas con discapacidad. Deben recibir información concerniente a sus cuerpos y a su vinculación con otras personas. Y tal educación debe servir para romper los estereotipos que les impiden ejercer su sexualidad y sus derechos en igualdad de condiciones.

Finalmente, una prestación del derecho a la educación que linda con el derecho al trabajo es la referida a la formación técnica de los NNA para lograr su empleabilidad el momento de su adultez, en particular para aquellos que cumplirán la mayoría de edad en un CAR. Sobre este punto, las Directrices de Naciones Unidas sobre las Modalidades Alternativas de Cuidado de Niños disponen lo siguiente: ${ }^{19}$

130. Las agencias y los centros de acogida deberían aplicar unas politicas claras y ejecutar los procedimientos acordados relativos a la conclusión programada o no de su trabajo con los niños con objeto de velar por la reinserción social o el seguimiento adecuados. Durante todo el período de acogida, dichas agencias y centros deberían fijarse sistemáticamente como objetivo la preparación del niño para asumir su independencia e integrarse plenamente en la comunidad, en particular su preparación para la vida cotidiana y el trato social, que se fomenta mediante la participación en la vida de la comunidad local.

131. El proceso de transición del acogimiento a la reinserción social debería tener en cuenta el género, la edad, la madurez y las circunstancias particulares del niño y comprender orientación y apoyo, en especial para evitar la explotación. Se debería alentar a los niños cuyo acogimiento llegue a su fin, a que participen en la planificación de su reinserción social. Los niños con necesidades especiales, como discapacidades, deberían poder acogerse a un sistema de asistencia apropiado, que entre otras cosas les permita eludir una institucionalización innecesaria. Debería alentarse a los sectores público y privado, incluso mediante incentivos, a emplear a niños de diferentes servicios de acogida, especialmente niños con necesidades especiales (parágrafos 130, 131).

Si bien la inclusión laboral de personas con discapacidad en nuestro país es más complicada, (Constantino \& Galicia, 2015, p. 261) no por ello se debe dejar de intentar lograr la máxima autonomía para este colectivo. Eso

19 Directrices sobre las Modalidades Alternativas de Cuidado de los Niños. Op. Cit., parágrafos 130 y 131 
significa, para el caso concreto, educar al niño o niña para conseguir la mayor autonomía posible en el futuro. Eso se traduce en lograr que pueda trabajar y recibir un sueldo. En ese sentido, la educación, al menos en los últimos años de la adolescencia, debe enfocarse en lograr una adecuada inclusión al mundo laboral. De otra manera, será difícil lograr el externamiento de estos niños y niñas cuando lleguen a la edad adulta, como fue mencionado páginas arriba.

\subsection{DERECHO AL JUEGO}

El artículo 31 de la CDN establece el derecho de niños y niñas al descanso y el esparcimiento, al juego y a las actividades recreativas propias de su edad. En el mismo sentido, las Directrices de Naciones Unidas sobre las Modalidades Alternativas de Cuidado de los Niños disponen que los acogedores deben velar por el respeto del derecho de niños y niñas a desarrollarse mediante el juego y las actividades de esparcimiento (parágrafo 85).

Esta dimensión de la niñez es significativa para su desarrollo emocional, por lo que no debe ser una tarea relegada en los CAR. Sin embargo, para el año 2010 la Defensoría del Pueblo había constatado que, en los CAR verificados por ella, solo el $4.5 \%$ desarrollaba actividades de juego (2010, p. 277). Esto quiere decir que hay una carencia para niños y niñas con y sin discapacidad.

No obstante, paraniñosyniñascondiscapacidadlafalta deaccesibilidad puede hacer todo esto mucho más complicado. Actividades comunes como ir al parque pueden generar exclusión para quienes no cuentan con la accesibilidad necesaria para acudir de manera sencilla. Ciertas actividades especiales como excursiones pueden terminar excluyendo a niños y niñas con discapacidad si se cree que es muy costoso o complicado llevarlos. Al respecto, debe tenerse en cuenta que, a partir de la Ley 30603, Ley que garantiza el derecho al juego y la accesibilidad urbana para niños, niñas y adolescentes ${ }^{20}$, se modificó la Ley General de la Persona con Discapacidad para indicar que "los espacios públicos y privados de uso público con fines recreacionales que cuenten con juegos infantiles, deben ser accesibles para niños, niñas y adolescentes con discapacidad y cumplir con los estándares de diseño universal." En ese sentido, los espacios de juego que se diseñen en cualquier lugar del país deben pensar en la accesibilidad de niños y niñas con discapacidad. De la misma forma, las actividades que se puedan diseñar para niños y niñas como paseos, fiestas, o excursiones deben tener en cuenta las posibilidades de participación de niños y niñas con discapacidad.

20 Publicada el 5 de julio de 2017. 


\section{CONCLUSIONES}

Niños y niñas se enfrentan a diversas situaciones discriminatorias y vulneradoras de derechos en diferentes ámbitos. La familia, que debería ser un espacio de protección y valoración, también puede ser un agente que no favorezca su desarrollo. Ante ello, el Estado debe tomar medidas. No obstante, estas deben tener en cuenta que la institucionalización es una medida de ultima ratio que puede tener graves consecuencias en niños y niñas.

En el caso de niños y niñas con discapacidad, la complejidad aumenta. La falta de apoyos sociales suele generar que se encuentren en situación de desprotección en más ocasiones. Y ante ello, las respuestas del Estado aún no se adecúan completamente a las necesidades de niños y niñas con discapacidad. En ese sentido, se requieren actuaciones con un enfoque de discapacidad que salvaguarden los derechos de estos niños y niñas y que fomenten su autonomía de manera que puedan convertirse en adultos y adultas que puedan ejercer su ciudadanía de manera plena.

\section{REFERENCIAS}

Aguerre, I., Riley-Powell, A., Weldon, C., Pajuelo, M., Celis Nacimento, R. A., Puente-Arnao, A., Cabrera, L., Oberhelman,R. \& Paz-Soldan, V. A. (2019). "Knocking on Doors that Don't Open": Experiences of caregivers of children living with disabilities in Iquitos and Lima, Peru. Disability and Rehabilitation, 41(21), 2538-2547. https: / / doi.org /10.1080/09638288.2018.1471741

Bernabe-Ortiz, A., Diez-Canseco, F., Vasquez, A., Kuper, H., Walsham, M., \& Blanchet, K. (2016). Inclusion of persons with disabilities in systems of social protection: A population-based survey and case-control study in Peru. BMJ Open, 6(8), e011300. https://doi.org/10.1136/ bmjopen-2016-011300

Bregaglio, R. (2104). Alcances del mandato de no discriminación en la Convención sobre los derechos de las personas con discapacidad. En E. Salmón y R. Bregaglio (Eds.). Nueve conceptos claves para entender la Convención sobre los derechos de las personas con discapacidad, pp. 73-97. IDEHPUCP.

Comité de Derechos del Niño. (2016). Observaciones finales sobre los informes periódicos cuarto y quinto combinados del Perú. CRC/C/PER/CO/4-5. 
Recuperado de https://www.mimp.gob.pe/webs/mimp/pnaia/ pdf/obs-finales-cuarto-quinto-combinados-2016.pdf.

Comité de Derechos Económicos, Sociales y Culturales. (1999). Observación General 13. El derecho a la educación (artículo13 del Pacto) E/C.12/1999/10. Recuperado de http://hrlibrary.umn.edu/gencomm/epcomm13s.htm.

Comité sobre los Derechos de las Personas con Discapacidad. (2017). Observación General núm. 5 (2017) sobre el derecho a vivir de forma independiente y a ser incluido en la comunidad. CRPD/C/GC/5.

Constantino, R. (2015). Rampas, camillas, pastillas y más: Barreras en el ejercicio del derechoala salud de personas con discapacidad. Recuperadode:http:/ / institutopaz.net/sistema/data / files / folleto_rampas $\% 2 \mathrm{C} \% 20$ camillas_pastillas_mas.pdf

Constantino, R. A., Bregaglio, R. A., \& Cueva, S. del P. (2016). Al final del salón: Un diagnóstico de la situación de la educación de personas con discapacidad en el Perú. Recuperado de: http://www.institutopaz.net/recursos / diagnostico-educacion-de-personas-discapacidad-peru

Constantino, R. A., \& Galicia, S. (2015). La configuración de los ajustes razonables en el ámbito laboral peruano: Definiciones, omisiones y propuestas. Anuario de Investigación del CICAJ 2013-2014. Las instituciones jurídicas en debate, p. 42.

Crenshaw, K. (1991). Mapping the Margins: Intersectionality, Identity Politics and violence Against Women of Color. Stanford Law Review, 43(6), pp. 1241-1299. doi: 10.2307/1229039

Del Águila, L. M. (2015). La autonomía de las personas con discapacidad como principio rector. E. Salmón y R. Bregaglio (Eds.). Nueve conceptos claves para entender la Convención sobre los derechos de las personas con discapacidad (51-72). IDEHPUCP.

Dörr, A. y Banz, C. (2010). Ventanas de oportunidades y capacidad de establecer vínculo afectivo en niños ferales $\mathrm{v} / \mathrm{s}$ niños abandonados en instituciones. Psiquiatría Universitaria, 6(1), pp. 38-45. Recuperado de http://www.revistagpu.cl/GPU\%201\%20(2010)/ENS\%20 Ventanas\%20de\%20oportunidad.pdf.

Defensoría del Pueblo. (2010). Informe Defensorial 150. El derecho de los niños, niñas y adolescentes a vivir en una familia: la situación de los Centros de 
Atención Residencial estatales desde la mirada de la Defensoría del Pueblo. Lima:Defensoría delPueblo.Recuperadodehttps: / / www.defensoria. gob.pe/wp-content/uploads/2018/05/informe-150-2010.pdf.

Defensoría del Pueblo. (2011). Informe Defensorial 153. Niños, niñas y adolescentes en abandono: aportes para un nuevo modelo de atención. Lima: Defensoría del Pueblo. Recuperado de https:/ / www.mimp.gob.pe/ webs/mimp/sispod/pdf/134.pdf.

Human Rights Watch. (2014). Abandoned by the State. Violence, Neglect, and Isolation for Children with Disabilities in Russian Orphanages [Reporte]. Recuperado de https://www.hrw.org/report/2014/09/15/ abandoned-state/violence-neglect-and-isolation-children-disabilitiesrussian.

Human Rights Watch. (2016). "It is My Dream to Leave This Place". Children with Disabilities in Serbian institutions. [Reporte]. Recuperado de https: / / www.hrw.org/report/2016/06/08/it-my-dream-leaveplace/children-disabilities-serbian-institutions.

Programa Integral Nacional para el Bienestar Familiar. (2017). Anuario Estadístico de la Población Atendida 2017. Ministerio de la Mujer y Poblaciones Vulnerables. Recuperado de https://www. inabif.gob.pe/portalweb / portal/portaldeestadisticas / 2017/ mensual/170_10_2017.pdf.

Minieri, S. (2017). Derechos sexuales y derechos reproductivos de las mujeres con discapacidad. Aportes teóricos para una agenda de incidencia inclusiva. Recuperado de: http://www.redaas.org.ar/archivos-recursos/401Derechos $\% 20$ sexuales $\% 20 y \% 20$ derechos $\% 20$ reproductivos $\% 20$ de $\% 201$ as $\% 20$ mujeres $\% 20$ con $\% 20$ discapacidad. $\% 20-\% 20$ Sofia $\% 20$ Minieri(Agosto\%202017).pdf

Mulheir, G. (2012). Deinstitutionalisation - A Human Rights Priority for Children with Disabilities. The Equal Rights Review, 9, pp. 117-137. Recuperado de https:/ /www.equalrightstrust.org/ertdocumentbank/ err9_mulheir.pdf.

Oliver, M., \& Barnes, C. (2012). The new politics of disablement. Palgrave Macmillan. 
Palacios, A. (2008). El modelo social de discapacidad: Orígenes, caracterización y plasmación en la Convención Internacional sobre los Derechos de las Personas con Discapacidad. Cinca y CERMI.

Sarmiento, A. J.; Puhl, S. M.; Oteyza, G.; Bermúdez, F.; Siderakis, M. (2009). Las conductas transgresoras de los adolescentes en conflicto con la ley penal: su relación con las perturbaciones en el vínculo primario. Anuario de Investigaciones, 16, 51-57. Recuperado de http://www. redalyc.org/pdf/3691/369139945041.pdf.

Siegel, L. (2014). The Human Right to Language: Communication Access for Deaf Children. Gallaudet University Press. Washington DC.

Tomasevski,K. (2001). Human rights obligations: making education available, accessible, acceptable and adaptable. Right to Education Primers No. 3. Gothenburg: Right to Education Initiative. Recuperado de https:/ / www.right-to-education.org/resource/primer-no-3-human-rightsobligations-making-education-available-accessible-acceptable-and.

UNICEF. (2014). Llamado a la acción. Poner fin al internamiento de niñas y niños menores de tres años en instituciones de protección. Recuperado de: http://www.unicef.org/ecuador/llamado_ala_accion_pdf.pdf.

Valega Chipoco, C. (2016). Barreras que enfrentan las personas en situación de discapacidad en el ejercicio de su sexualidad y autodeterminación reproductiva. La manzana de la discordia, julio - diciembre, 2016 Vol. 11, No. 2.

Walzer, M. (2001). Las esferas de la justicia. Una defensa del pluralismo y la igualdad. (H. Rubio, Trad.). Fondo de Cultura Económica.

\section{INSTRUMENTOS JURÍDICOS}

Convención sobre los Derechos del Niño. Resolución 44/25 de la Asamblea General de Naciones Unidas, de 20 de noviembre de 1989.

Directivas de Naciones Unidas sobre las Modalidades Alternativas de Cuidado de los Niños. Resolución 64/142 de la Asamblea General de Naciones Unidas, de 24 de febrero de 2010. 
Decreto Legislativo 1297, Decreto Legislativo para la protección de niñas, niños y adolescentes sin cuidados parentales o en riesgo de perderlos. Publicado en el Diario Oficial El Peruano el 30 de diciembre de 2016.

Decreto Supremo 008-2010-SA, Reglamento de la Ley 29344 - Ley Marco de Aseguramiento Universal en Salud. Publicado en el Diario Oficial El Peruano el 3 de abril de 2010.

Decreto Legislativo 1164, Decreto Legislativo que establece disposiciones para la extensión de la cobertura poblacional del Seguro Integral de Salud en materia de Afiliación al Régimen de Financiamiento Subsidiado. Publicado en el Diario Oficial El Peruano el 7 de diciembre de 2013.

Ley 30603, Ley que garantiza el derecho al juego y la accesibilidad urbana para niños, niñas y adolescentes. Publicada en el Diario Oficial El Peruano el 5 de julio de 2017

Manual de Acreditación y Supervisión de Programas para Niños, Niñas y Adolescentes Sin Cuidados Parentales. Aprobado mediante Resolución Ministerial 081-2012-MIMP del 4 de abril de 2012.

Manual de Intervención en Centros de Atención Residencial de Niños, Niñas y Adolescentes Sin Cuidados Parentales. Aprobado mediante Resolución Ministerial 080-2012-MIMP del 4 de abril de 2012.

Manual para la Atención Integral y la Prevención del Abandono de las Personas con Discapacidad. Aprobado mediante Resolución de INABIF 107-2015, del 26 de febrero de 2015.

Tribunal Constitucional del Perú. (2004). Sentencia recaída en el expediente 2868-2004-AA/TC.

Fecha de recepción: 31 de agosto de 2019

Fecha de aceptación: 13 de noviembre de 2019 